\title{
THE SOFTWARE TOOLWORKS OPEN CHAMPIONSHIP
}

\author{
Feng-hsiung Hsu \\ Carnegie-Mellon University \\ Pittsburgh, Pennsylvania
}

Deep Thought tied for first place with GM Miles in the open section of the Software Toolworks Chess Championship held in Long Beach, CA. in November 1988. Over 700 players were attracted by the $\$ 130.000$ total prize fund, 76 of them (mainly rated over 2300) in the open section, including former world champion GM Mikhail Tal, the "Great Dane" GM Bent Larsen, GM Samuel Reshevsky, GM Walter Browne, GM Gurevich, GM Gruefeld, GM Tony Miles and several IMs. Deep Thought ended with a score of 6.5 out of 8 . Deep Thought's opponents were:

$\begin{array}{llc} & \text { USCF rating } & \text { Deep Thought's score } \\ \text { LeSiege } & 2334 & 1.0 \\ \text { Glicksman } & 2388 & 1.0 \\ \text { Bent Larsen (GM) } & 2590 & 1.0 \\ \text { Walter Browne (GM) } & 2640 & 0.0 \\ \text { McCambridge(IM) } & 2599 & 0.5 \\ \text { Salgado (FM) } & 2388 & 1.0 \\ \text { Fishbein (FM) } & 2572 & 1.0 \\ \text { Silman (IM) } & 2507 & 1.0\end{array}$

Deep Thought achieved a performance rating of 2745 in this tournament, breaking the previous record for computers (set by Deep Thought itself) by over 100 points. GM Bent Larsen had the misfortune to find his name becoming the answer to the Trivial Pursuit question "Who is the first GM to lose to a computer under regular time control in a tournament?" by his third-round loss to Deep Thought.

Deep Thought's newly established rating is estimated at over 2545 .

Six-month old Deep Thought has now played 42 rated games. It played against International Masters 7 times, won 5 and drew 2. It played International Grand Masters 3 times, winning 1 and losing 2 (the loss against GM Lev Alburt was due to a bug that caused the machine to throw away a repetition draw).

Deep Thought has now met the qualificatons for the Fredkin Intermediate Prize for the first computer with a rating of over 2500 in 25 consecutive games.

The Deep Thought team would like to take this opportunity to thank all those whose help and encouragement made this possible, particularly Lawrence Butcher, Stuart Cracraft, Jim Gillogly, Peter Jansen, Larry Kaufmann, Kau-Fu Lee, Tom Mitchel, Raj Reddy, Danny Sleator, Ken Thompson, Hide Tokuda, John Zsarnay and our advisors, Roberto Bisiani, Ed Clarke, H.T. Kund and Bob Sproull.

We would also like to give our special thanks to Stuart Cracraft for spending his Thanksgiving Weekend operating Deep Thought on site.

The Deep Thought team includes: Thomas Anantharaman, Mike Browne, Murray Campbell, Feng-hsiung Hsu and Andreas Notwatzyk, all with the Computer Science Department at Carnegie-Mellon University.

Some of the more interesting games follow. 
Deep Thought $v s$. Glicksman (2388), round 2

1. e4 e6 2. d4 d5 3. Nc3 Bb4 4. e5 Ne7 5. Bd2 c5 6. Nb5 Bxd2 7. Qxd2 Nf5 8. dxc5 a6 9. Nd6 Nxd6 10. cxd6 Nc6 11. f4 f6 12. Nf3 0-0 13. Be2 fxe5 14. fxe5 Rf5 15. Qc3 d4 16. Qd2 Nxe5 17. Nxd4 Qh4 18. g3 Qe4 19. 0-0-0 Rf2 20. Rhe1 Bd7 21. Qc3 Rc8 22. Bc4 Qg2 23. Rxe5 b5 24. Bxe6 Bxe6 25. Rc5 Rxc5 26. Qxc5 Bg4 27. Qc6 Rf1 28. Qe8 Rf8 29. d7 Qd5 30. Rf1 Black resigns.

White announced mate in 19 moves (37 plies).

GM Bent Larsen (FIDE 2560) vs. Deep Thought, round 3

1. c4 e5 2. g3 Nf6 3. Bg2 c6 4. Nf3 e4 5. Nd4 d5 6. cxd5 Qxd5 7. Nc2 Qh5 8. h4 Bf5 9. Ne3 Bc5 10. Qb3 b6 11. Qa4 0-0 12. Nc3 b5 13. Qc2 Bxe3 14. dxe3 Re8 15. a4 b4 16. Nb1 Nbd7 17. Nd2 Re6 18. b3 Rd8 19. Bb2 Bg6 20. Nc4 Nd5 21. 0-0-0 N7f6 22. Bh3 Bf5 23. Bxf5 Qxf5 24. f3 h5 25. Bd4 Rd7 26. Kb2 Rc7 27. g4 hxg4 28. Rhg1 c5 29. fxg4 Nxg4 30. Bxg7 Rg6 31. Qd2 Rd7 32. Rxg4 Rxg4 33. Ne5 Nxe3 34. Qxd7 Nxd1 35. Qxd1 Rg3 36. Qd6 Kxg7 37. Nd7 Re3 38. Qh2 Kh7 39. Nf8 Kh8 40. h5 Qd5 41. Ng6 fxg6 42. hxg6 Kg7 43. Qh7 Kf6 White resigns.

\section{Deep Thought $v$ s. GM Walter Browne (2640), round 4}

1. e4 c5 2. c3 Nf6 3. e5 Nd5 4. d4 cxd4 5. Nf3 Nc6 6. Bc4 Nb6 7. Bb3 d5 8. exd6 e.p. Qxd6 9. 0-0 e6 10. cxd4 Be7 11. Nc3 0-0 12. Rfe1 Nd5 13. g3(?) Qd8 14. a3 Nxc3 15. bxc3 b6 16. Qd3 Bb7 17. Bc2 g6 18. Bf4 Rac8 19. Bh6 Rfe8 20. Bd2 Na5 21. Ba4 Qd5 22. Bxe8 Rxe8 23. Kg2 Nc4 24. Bc1 g5 25. h3 h5 26. g4 e5 27. Qd1 f5 28. gxh5 g4 29. hxg4 fxg4 30. Kg1 Qxf3 31. Qxf3 Bxf3 32. Bh6 Kh7 33. Bd2 Ref8 34. Rxe5 Nxe5 35. Re1 Nc6 White resigns.

The following game is the second time that Deep Thought won a KRBKR ending.

Deep Thought $v s$. FM Alex Fishbein (2572), round 7

1. e4 e5 2. Nf3 Nc6 3. Bb5 a6 4. Bxc6 dxc6 5. 0-0 f6 6. d4 exd4 7. Nxd4 c5 8. Ne2 Qxd1 9. Rxd1 Bd7 10. Bf4 0-0-0 11. c4 Ne7 12. Nc3 Re8 13. Be3 Nc6 14. Rd2 Ne5 15. b3 h5 16. h3 b6 17. Nd5 Bc6 18. a4 a5 19. N2c3 Kb7 20. Nb5 Bxb5 21. axb5 Bd6 22. f3 Ra8 23. f4 Nf7 24. Kf2 Rhe8 25. Kf3 Bf8 26. Nc3 Nd6 27. Rd5 g6 28. g4 hxg4 29. hxg4 Bg7 30. f5 gxf5 31. gxf5 Rh8 32. Bf4 Rh3 33. Bg3 Rg8 34. Rd3 Bf8 35. Kg2 Rh4 36. Re1 Rhg4 37. Kh3 a4 38. bxa4 R8g7 39. Bh4 Nxc4 40. Bxf6 Rg8 41. e5 Bh6 42. Nd5 Bf4 43. Rf3 Bxe5 44. Rxe5 Nxe5 45. Bxe5 R4g5 46. Bg3 c4 47. Nc3 Rh5 48. Kg2 R8g5 49. f6 Rf5 50. Re3 Rxf6 51. Re7 Rff5 52. Rxc7 Ka8 53. a5 Rxb5 54. Nxb5 Rxb5 55. axb6 Rxb6 56. Bf4 Rb3 57. Kf2 Rb7 58. Rxc4 Rb2 59. Ke3 Kb7 60. Ke4 Rb5 61. Be3 Rh5 62. Bd4 Rg5 63. Be5 Kb6 64. Kd5 Kb5 65. Rc8 Rg6 66. Rc5 Kb6 67. Rc2 Rh6 68. Rb2 Ka6 69. Bd6 Rh5 (sealed move) 70. Kc6 Rg5 71. Rb3 Rh5 72. Rb1 Rg5 73. Rb2 Rh5 74. Rb8 Rh7 75. Bf8 Ka7 76. Rb3 Ka8 77. Re3 Rb7 78. Re4 Rf7 79. Re8 Ka7 80. Bc5 Ka6 81. Ra8 Black resigns.

Mephisto four times World Champion, but the Spracklens hold the same record.

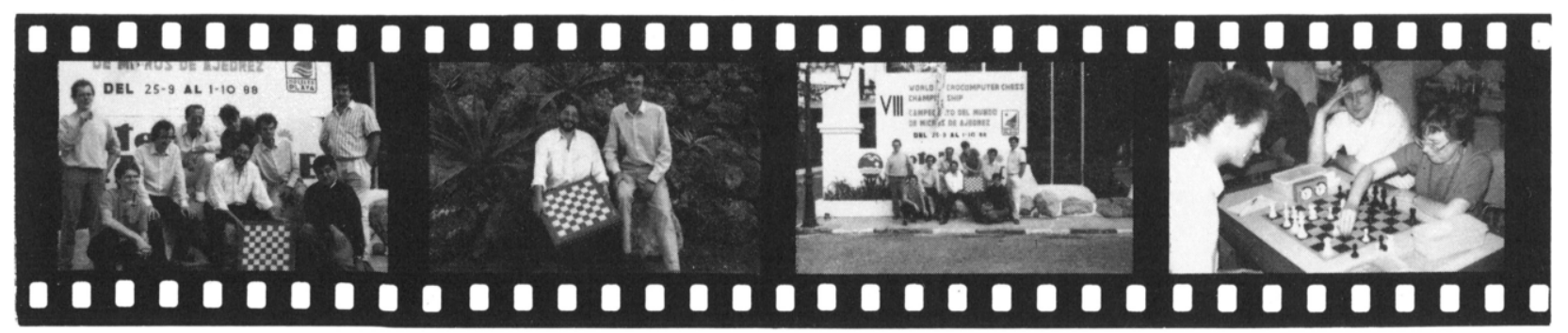

Photo bij Frederic Friedel and Dieter Steinwender 